\title{
Periodic solutions for Liénard type equation with time-variable coefficient
}

Fabao Gao ${ }^{1,2^{*}}$, Shiping $\mathrm{Lu}^{3}$ and Minghui Yao ${ }^{4}$

"Correspondence:

gaofabao@sina.com;

gaofabao@vt.edu

'School of Mathematical Science,

Yangzhou University, Yangzhou, 225002, China

${ }^{2}$ Department of Engineering Science and Mechanics, Virginia Tech, Blacksburg, VA 24061, USA Full list of author information is available at the end of the article

\begin{abstract}
By means of topological theory, a class of Liénard type equations with a deviating argument of the form $\left(\varphi_{p}\left(x^{\prime}(t)\right)\right)^{\prime}+f(x(t-\tau)) x^{\prime}(t-\tau)+\beta(t) g(x(t-\tau))=e(t)$ is studied. It is notable that the coefficient $\beta(t)$ in front of $g(x(t-\tau))$ is allowed to change sign in this paper. Moreover, a numerical simulation is carried out to verify the validity of the obtained results. In addition, the generalized form of the above equation with time-varying delays is also discussed briefly.
\end{abstract}

Keywords: periodic solution; $p$-Laplacian; topological theory; time-varying coefficient

\section{Introduction}

Consider the Liénard type $p$-Laplacian differential equation

$$
\left(\varphi_{p}\left(x^{\prime}(t)\right)\right)^{\prime}+f(x(t-\tau)) x^{\prime}(t-\tau)+\beta(t) g(x(t-\tau))=e(t),
$$

where $p>1$ and $\varphi_{p}: R \mapsto R$ is given by $\varphi_{p}(s)=|s|^{p-2} s$ for $s \neq 0$ and $\varphi_{p}(0)=0, \int_{0}^{T} \beta(s) d s \neq 0$, $f, g, e \in C(R, R)$, and $e(t)$ is $T$-periodic, $\int_{0}^{T} e(s) d s=0, T, \tau>0$ are given constants. The equation can be applied to the dynamics of fluids. An example is Euler's equation, governing the flow of an ideal fluid in a conservative force field. While there are plenty of results on the existence of periodic solutions for the $p$-Laplacian equation (see [1-10] and references therein), studying delay Liénard equations with a variable coefficient in front of the nonlinear term is relatively uncommon. The main difficulty lies in finding a priori bounds for (1.1) requiring the coefficient of the nonlinear term to keep a fixed sign.

In the aforementioned literature, based on the Leray-Schauder degree theory, Amster et al. [1] considered the existence of at least one periodic solution for the $p$-Laplacian-like system with a fixed delay. Gao and Zhang [2] discussed an $n$-dimensional $p$-Laplacian-like neutral functional differential equation, and they established some criteria to guarantee the existence of periodic solutions for the equation by using Mawhin's continuation theorem. Manásevich and Sędziwy [3] were concerned with the existence and uniqueness of a limit circle for a generalized Liénard type equation, which involves the one-dimensional $p$-Laplacian operator and a positive small parameter. Manásevich and Mawhin [4] studied the existence of periodic solutions to some system cases involving the fairly general vector-valued $p$-Laplacian operator. Aizicovici et al. [5] investigated a nonlinear periodic

(c) 2015 Gao et al.: licensee Springer. This article is distributed under the terms of the Creative Commons Attribution 4.0 International License (http://creativecommons.org/licenses/by/4.0/), which permits unrestricted use, distribution, and reproduction in any medium, provided you give appropriate credit to the original author(s) and the source, provide a link to the Creative Commons license, and indicate if changes were made. 
problem driven by the scalar $p$-Laplacian with a nonsmooth potential via the degree map; they proved the existence of at least three distinct nontrivial solutions, two of which have a constant sign. In Gaines and Mawhin's monograph [6], coincidence degree theory was described and used to study alternative problems. Aizicovici et al. [7] conducted periodic problems driven by the scalar $p$-Laplacian with a multivalued right-hand side nonlinearity. Gao et al. [8, 9] considered the existence of periodic solutions for two kinds of Rayleigh type $p$-Laplacian equations by using the continuation theorem. Cheung [10] proposed the existence of periodic solutions of a $p$-Laplacian Rayleigh equation with two deviating arguments.

Based on topological theory and some analysis techniques, the existence of periodic solutions for (1.1) is investigated in the present paper. It is significant that the coefficient $\beta(t)$ of the nonlinear term can change sign, which cannot be achieved in most of the previous papers. Furthermore, a numerical simulation is performed to validate the feasibility of the obtained results. In addition, the fixed delays in (1.1) are also extended to the time-varying delays and we briefly discuss them. Moreover, the approaches used to estimate a priori bounds of periodic solutions are different from the corresponding ones in the literature.

\section{Preliminaries}

Let us consider the problem: find $u \in C_{T}^{1}$ such that

$$
\left(\varphi_{p}\left(u^{\prime}(t)\right)\right)^{\prime}=\widetilde{f}\left(t, u(t-\tau), u^{\prime}(t-\tau)\right) .
$$

Lemma 2.1 (see Amster et al. [1]) Let $\Omega \in C_{T}^{1}$ be an open set. Assume that:

$\left(\mathrm{A}_{1}\right)$ For $\lambda \in(0,1]$ the problem

$$
\left(\varphi_{p}\left(u^{\prime}(t)\right)\right)^{\prime}=\lambda \tilde{f}\left(t, u(t-\tau), u^{\prime}(t-\tau)\right)
$$

has no solution on $\partial \Omega$.

$\left(\mathrm{A}_{2}\right)$ The equation

$$
\widetilde{F}(a) \triangleq \frac{1}{T} \int_{0}^{T} \widetilde{f}(t, a, 0)=0
$$

has no solution on $\partial \Omega \cap R^{n}$.

$\left(\mathrm{A}_{3}\right)$ The Brouwer degree

$$
\operatorname{deg}\left(\widetilde{F}, \Omega \cap R^{n}, 0\right) \neq 0 .
$$

Then the problem (2.1) has at least one solution in $C_{T}^{1}$.

\section{Main results}

For the sake of convenience, we only study the periodic solutions of (1.1) in the case $\int_{0}^{T} \beta(t) d t>0\left(\int_{0}^{T} \beta(t) d t<0\right.$ can be discussed in the same way).

Theorem 3.1 Assume that the following conditions hold: 
$\left(\mathrm{H}_{1}\right)$ There are positive constants $m_{1}, m_{2}$, and $d$ such that

$$
m_{1}|u|^{p-1} \leq|g(u)| \leq m_{2}|u|^{p-1}, \quad \forall|u|>d
$$

$\left(\mathrm{H}_{2}\right)$ There is a constant $r \geq 0$ such that $\lim _{|u| \rightarrow \infty} \frac{|F(u)|}{|u|^{p-1}} \leq r$, where $F(x)=\int_{0}^{x} f(s) d s$.

$\left(\mathrm{H}_{3}\right)$

$$
A:= \begin{cases}{\left[\frac{\left(\beta_{\infty}+\varepsilon_{1}\right) m_{2} T}{m_{1} \int_{0}^{T}\left(\beta(t)+\beta_{\infty}+\varepsilon_{1}\right) d t}\right]^{\frac{1}{p-1}} 2^{\frac{2-p}{p-1}}<1,} & 1<p \leq 2, \\ {\left[\frac{\left(\beta_{\infty}+\varepsilon_{1}\right) m_{2} T}{m_{1} \int_{0}^{T}\left(\beta(t)+\beta_{\infty}+\varepsilon_{1}\right) d t}\right]^{\frac{1}{p-1}}<1,} & p>2,\end{cases}
$$

where $\varepsilon_{1}>0, \beta_{\infty}=\max _{t \in[0, T]}|\beta(t)|$.

$\left(\mathrm{H}_{4}\right) \quad u g(u)>0, \forall|u|>d$.

Then (1.1) has at least one T-periodic solution, if $C_{p} r\left[\frac{T}{2(1-A)}\right]^{p-1}+\frac{\beta_{\infty} m_{2} T}{2(1-A)^{p}}<1$, where

$$
C_{p}= \begin{cases}1, & 1<p \leq 2, \\ 2^{p-2}, & p>2\end{cases}
$$

Proof Consider the homotopic equation of (1.1) as follows:

$$
\left(\varphi_{p}\left(x^{\prime}(t)\right)\right)^{\prime}+\lambda f(x(t-\tau)) x^{\prime}(t-\tau)+\lambda \beta(t) g(x(t-\tau))=\lambda e(t), \quad \lambda \in(0,1] .
$$

For $\forall \varepsilon_{1}>0, \beta_{\infty}=\max _{t \in[0, T]}|\beta(t)|$, (3.1) can be written in the following form:

$$
\begin{aligned}
& \left(\varphi_{p}\left(x^{\prime}(t)\right)\right)^{\prime}+\lambda f(x(t-\tau)) x^{\prime}(t-\tau)+\lambda\left(\beta(t)+\beta_{\infty}+\varepsilon_{1}\right) g(x(t-\tau)) \\
& =\lambda\left(\beta_{\infty}+\varepsilon_{1}\right) g(x(t-\tau))+\lambda e(t), \quad \lambda \in(0,1] .
\end{aligned}
$$

Integrating both sides of (3.2) from 0 to $T$ and using the integral mean value theorem, there exists a constant $\xi \in(0, T)$ such that

$$
g(x(\xi-\tau)) \int_{0}^{T}\left(\beta(t)+\beta_{\infty}+\varepsilon_{1}\right) d t=\int_{0}^{T}\left(\beta_{\infty}+\varepsilon_{1}\right) g(x(t-\tau)) d t .
$$

Now, we claim that

$$
|x(\xi-\tau)| \leq A|x|_{\infty}+B,
$$

where

$$
\begin{aligned}
& A= \begin{cases}{\left[\frac{\left(\beta_{\infty}+\varepsilon_{1}\right) m_{2} T}{m_{1} \int_{0}^{T}\left(\beta(t)+\beta_{\infty}+\varepsilon_{1}\right) d t}\right]^{\frac{1}{p-1}} 2^{\frac{2-p}{p-1}},} & 1<p \leq 2, \\
{\left[\frac{\left(\beta_{\infty}+\varepsilon_{1}\right) m_{2} T}{m_{1} \int_{0}^{T}\left(\beta(t)+\beta_{\infty}+\varepsilon_{1}\right) d t}\right]^{\frac{1}{p-1}},} & p>2,\end{cases} \\
& B= \begin{cases}{\left[\frac{\left(\beta_{\infty}+\varepsilon_{1}\right) M_{g} T}{m_{1} \int_{0}^{T}\left(\beta(t)+\beta_{\infty}+\varepsilon_{1}\right) d t}\right]^{\frac{1}{p-1}} 2^{\frac{2-p}{p-1}}+d,} & 1<p \leq 2, \\
{\left[\frac{\left(\beta_{\infty}+\varepsilon_{1}\right) M_{g} T}{m_{1} \int_{0}^{T}\left(\beta(t)+\beta_{\infty}+\varepsilon_{1}\right) d t}\right]^{\frac{1}{p-1}}+d,} & p>2,\end{cases} \\
& M_{g}=\max _{|u| \leq d}|g(u)| .
\end{aligned}
$$


Gao et al. Advances in Difference Equations (2015) 2015:125

Page 4 of 9

Case 1: If $|x(\xi-\tau)| \leq d$, then (3.4) holds clearly.

Case 2: If $|x(\xi-\tau)|>d$. Denote

$$
E_{1}=\{t: t \in[0, T],|x(t-\tau)| \leq d\}, \quad E_{2}=\{t: t \in[0, T],|x(t-\tau)|>d\} .
$$

From (3.3), we have

$$
|g(x(\xi-\tau))| \int_{0}^{T}\left(\beta(t)+\beta_{\infty}+\varepsilon_{1}\right) d t \leq\left(\beta_{\infty}+\varepsilon_{1}\right)\left(\int_{E_{1}}+\int_{E_{2}}\right)|g(x(t-\tau))| d t,
$$

which, together with assumption $\left(\mathrm{H}_{1}\right)$, leads to

$$
\begin{aligned}
|x(\xi-\tau)|^{p-1} m_{1} \int_{0}^{T}\left(\beta(t)+\beta_{\infty}+\varepsilon_{1}\right) d t & \leq\left(\beta_{\infty}+\varepsilon_{1}\right)\left(\int_{E_{2}} m_{2}|x(t-\tau)|^{p-1} d t+M_{g} T\right) \\
& \leq\left(\beta_{\infty}+\varepsilon_{1}\right) m_{2} T|x|_{\infty}^{p-1}+\left(\beta_{\infty}+\varepsilon_{1}\right) M_{g} T
\end{aligned}
$$

ie.,

$$
\begin{aligned}
|x(\xi-\tau)| \leq & {\left[\frac{\left(\beta_{\infty}+\varepsilon_{1}\right) m_{2} T}{m_{1} \int_{0}^{T}\left(\beta(t)+\beta_{\infty}+\varepsilon_{1}\right) d t}\right]^{\frac{1}{p-1}} 2^{\frac{2-p}{p-1}}|x|_{\infty} } \\
& +\left[\frac{\left(\beta_{\infty}+\varepsilon_{1}\right) M_{g} T}{m_{1} \int_{0}^{T}\left(\beta(t)+\beta_{\infty}+\varepsilon_{1}\right) d t}\right]^{\frac{1}{p-1}} 2^{\frac{2-p}{p-1}}, \quad 1<p \leq 2, \\
|x(\xi-\tau)| \leq & {\left[\frac{\left(\beta_{\infty}+\varepsilon_{1}\right) m_{2} T}{m_{1} \int_{0}^{T}\left(\beta(t)+\beta_{\infty}+\varepsilon_{1}\right) d t}\right]^{\frac{1}{p-1}}|x|_{\infty} } \\
& +\left[\frac{\left(\beta_{\infty}+\varepsilon_{1}\right) M_{g} T}{m_{1} \int_{0}^{T}\left(\beta(t)+\beta_{\infty}+\varepsilon_{1}\right) d t}\right]^{\frac{1}{p-1}}, \quad p>2 .
\end{aligned}
$$

Thus, from Case 1 and Case 2, we see that (3.4) holds.

Let $\xi-\tau=k T+\bar{\xi}$, where $k$ is an integer and $\bar{\xi} \in[0, T]$; noticing (3.4), we get

$$
|x(t)| \leq A|x|_{\infty}+B+\int_{\bar{\xi}}^{t}\left|x^{\prime}(s)\right| d s, \quad t \in[\bar{\xi}, \bar{\xi}+T]
$$

and

$$
|x(t-T)| \leq A|x|_{\infty}+B+\int_{t-T}^{\bar{\xi}}\left|x^{\prime}(s)\right| d s, \quad t \in[\bar{\xi}, \bar{\xi}+T] .
$$

Combining the above two inequalities, we obtain

$$
\begin{aligned}
|x|_{\infty} & =\max _{t \in[\bar{\xi}, \bar{\xi}+T]}|x(t)| \\
& \leq \max _{t \in[\bar{\xi}, \bar{\xi}+T]}\left\{A|x|_{\infty}+B+\frac{1}{2}\left(\int_{\bar{\xi}}^{t}\left|x^{\prime}(s)\right| d s+\int_{t-T}^{\bar{\xi}}\left|x^{\prime}(s)\right| d s\right)\right\} \\
& \leq A|x|_{\infty}+B+\frac{1}{2} \int_{0}^{T}\left|x^{\prime}(s)\right| d s .
\end{aligned}
$$


In view of $\left(\mathrm{H}_{3}\right)$, we have

$$
|x|_{\infty} \leq \frac{B}{1-A}+\frac{\int_{0}^{T}\left|x^{\prime}(s)\right| d s}{2(1-A)}
$$

On the other hand, noticing $C_{p} r\left[\frac{T}{2(1-A)}\right]^{p-1}+\frac{\beta_{\infty} m_{2} T}{2(1-A)^{p}}<1$, we easily see that there is a sufficiently small constant $\varepsilon_{2}>0$ such that

$$
C_{p}\left(r+\varepsilon_{2}\right)\left[\frac{T}{2(1-A)}\right]^{p-1}+\frac{\beta_{\infty} m_{2} T}{2(1-A)^{p}}<1 .
$$

By assumption $\left(\mathrm{H}_{2}\right)$ and for such $\varepsilon_{2}$, we know there exists a constant $\rho>d$ (independent of $\lambda$ ) such that

$$
|F(u)| \leq\left(r+\varepsilon_{2}\right)|u|^{p-1}, \quad|u|>\rho .
$$

Let

$$
\Delta_{1}=\{t: t \in[0, T],|x(t-\tau)| \leq \rho\}, \quad \Delta_{2}=\{t: t \in[0, T],|x(t-\tau)|>\rho\} .
$$

Multiplying both sides of (3.1) by $x(t)$ and integrating them with $[0, T]$, noticing (3.7), we get

$$
\begin{aligned}
\int_{0}^{T}\left|x^{\prime}(t)\right|^{p} d t= & -\int_{0}^{T}\left(\varphi_{p}\left(x^{\prime}(t)\right)\right)^{\prime} x(t) d t \\
= & \lambda \int_{0}^{T} f(x(t-\tau)) x^{\prime}(t-\tau) x(t) d t \\
& +\lambda \int_{0}^{T} \beta(t) g(t, x(t-\tau)) x(t) d t-\lambda \int_{0}^{T} e(t) x(t) d t \\
\leq & \left(\int_{\Delta_{1}}+\int_{\Delta_{2}}\right)\left|F(x(t-\tau)) x^{\prime}(t)\right| d t \\
& +\beta_{\infty}|x|_{\infty}\left(\int_{E_{1}}+\int_{E_{2}}\right)|g(x(t-\tau))| d t+e_{\infty}|x|_{\infty} T \\
\leq & {\left[M_{\rho}+\left(r+\varepsilon_{2}\right)|x|_{\infty}^{p-1}\right] \int_{0}^{T}\left|x^{\prime}(t)\right| d t+\beta_{\infty} m_{2} T|x|_{\infty}^{p} } \\
& +\beta_{\infty} M_{g} T|x|_{\infty}+e_{\infty} T|x|_{\infty},
\end{aligned}
$$

where $M_{\rho}=\max _{|u| \leq \rho}|F(u)|, e_{\infty}=\max _{t \in[0, T]}|e(t)|$.

Substituting (3.5) into (3.8) yields

$$
\begin{aligned}
\int_{0}^{T}\left|x^{\prime}(t)\right|^{p} d t \leq & \left(r+\varepsilon_{2}\right)\left[\frac{B}{1-A}+\frac{\int_{0}^{T}\left|x^{\prime}(t)\right| d t}{2(1-A)}\right]^{p-1} \int_{0}^{T}\left|x^{\prime}(t)\right| d t \\
& +\beta_{\infty} m_{2} T\left[\frac{B}{1-A}+\frac{\int_{0}^{T}\left|x^{\prime}(t)\right| d t}{2(1-A)}\right]^{p} \\
& +\left[M_{\rho}+\frac{\beta_{\infty} M_{g} T}{2(1-A)}+\frac{e_{\infty} T}{2(1-A)}\right] \int_{0}^{T}\left|x^{\prime}(t)\right|^{p} d t
\end{aligned}
$$




$$
\begin{aligned}
& +\left(\beta_{\infty} M_{g}+e_{\infty}\right) \frac{B T}{1-A} \\
\leq & C_{p}\left(r+\varepsilon_{2}\right)\left[\left(\frac{B}{1-A}\right)^{p-1}+\left(\frac{\int_{0}^{T}\left|x^{\prime}(t)\right| d t}{2(1-A)}\right)^{p-1}\right] \int_{0}^{T}\left|x^{\prime}(t)\right| d t \\
+ & \frac{\beta_{\infty} m_{2} T}{2(1-A)^{p}}\left(\int_{0}^{T}\left|x^{\prime}(t)\right| d t\right)^{p} \\
+ & {\left[M_{\rho}+\frac{\beta_{\infty} M_{g} T}{2(1-A)}+\frac{e_{\infty} T}{2(1-A)}\right] T^{\frac{1}{q}}\left(\int_{0}^{T}\left|x^{\prime}(t)\right|^{p} d t\right)^{\frac{1}{p}} } \\
+ & \left(\beta_{\infty} M_{g}+e_{\infty}\right) \frac{B T}{1-A}+\frac{2^{p-1} \beta_{\infty} m_{2} B^{p} T}{(1-A)^{p}} \\
& \leq\left[C_{p}\left(r+\varepsilon_{2}\right)\left[\frac{T}{2(1-A)}\right]^{p-1}+\frac{\beta_{\infty} m_{2} T}{2(1-A)^{p}}\right] \int_{0}^{T}\left|x^{\prime}(t)\right|^{p} d t \\
+ & \theta_{1}\left(\int_{0}^{T}\left|x^{\prime}(t)\right|^{p} d t\right)^{\frac{1}{p}}+\theta_{2},
\end{aligned}
$$

where

$$
\begin{aligned}
& C_{p}= \begin{cases}1, & 1<p \leq 2, \\
2^{p-2}, & p>2,\end{cases} \\
& \theta_{1}=C_{p}\left(r+\varepsilon_{2}\right)\left(\frac{B}{1-A}\right)^{p-1} T^{\frac{1}{q}}+\left[M_{\rho}+\frac{\beta_{\infty} M_{g} T}{2(1-A)}+\frac{e_{\infty} T}{2(1-A)}\right] T^{\frac{1}{q}}, \\
& \theta_{2}=\left(\beta_{\infty} M_{g}+e_{\infty}\right) \frac{B T}{1-A}+\frac{2^{p-1} \beta_{\infty} m_{2} B^{p} T}{(1-A)^{p}}, \\
& e_{\infty}=\max _{t \in[0, T]}|e(t)| .
\end{aligned}
$$

In view of (3.6) and $\frac{1}{p}<1$, it follows from (3.9) that there is a constant $M>0$ such that

$$
\int_{0}^{T}\left|x^{\prime}(t)\right|^{p} d t \leq M
$$

which implies that there exists a constant $M_{1}>0$ such that

$$
\left|x^{\prime}\right|_{\infty} \leq M_{1}
$$

From (3.5) and (3.10), we can see that there exists a constant $M_{2}$ such that

$$
|x|_{\infty} \leq \frac{B}{1-A}+\frac{T^{\frac{1}{q}} M^{\frac{1}{p}}}{2(1-A)} \triangleq M_{2} .
$$

According to (3.11) and (3.12), set $\Omega=\left\{x:\left|x^{\prime}\right|_{\infty}<M_{1}+1,|x|_{\infty}<M_{2}+1\right\}$, then we see that (1.1) has no solution on $\partial \Omega$ for $\lambda \in(0,1]$, and when $x(t)=M_{2}+1$ or $-M_{2}-1$, from $\left(\mathrm{H}_{4}\right)$, we can get

$$
-\frac{1}{T} \int_{0}^{T} \beta(t) g\left(M_{2}+1\right) d t<0,
$$




$$
-\frac{1}{T} \int_{0}^{T} \beta(t) g\left(-M_{2}-1\right) d t<0
$$

So, condition $\left(\mathrm{A}_{2}\right)$ of Lemma 2.1 is also satisfied.

Denote

$$
H(x, \mu)=\mu x+\frac{1-\mu}{T} \int_{0}^{T} \beta(t) g(x) d t
$$

and when $x \in \partial \Omega \cap R, \mu \in[0,1]$, in view of $\int_{0}^{T} \beta(s) d s>0$, we have

$$
x H(x, \mu)=\mu x^{2}+\frac{(1-\mu) x}{T} \int_{0}^{T} \beta(t) g(x) d t>0 .
$$

Thus, $H(x, \mu)$ is a homotopic transformation and

$$
\begin{aligned}
\operatorname{deg}\{\widetilde{F}, \Omega \cap R, 0\} & =\operatorname{deg}\left\{-\frac{1}{T} \int_{0}^{T} \beta(t) g(x) d t, \Omega \cap R, 0\right\} \\
& =\operatorname{deg}\{x, \Omega \cap R, 0\} \neq 0 .
\end{aligned}
$$

Therefore, condition $\left(\mathrm{A}_{3}\right)$ of Lemma 2.1 is also satisfied. By using Lemma 2.1, we conclude that (1.1) has at least one $T$-periodic solution $x(t)$ on $\bar{\Omega}$ with $|x|_{\infty} \leq M_{2}$. This completes the proof of Theorem 3.1.

\section{Generalization}

As a matter of fact, (1.1) can also be extended to the time-varying case, which admits the following form:

$$
\left(\varphi_{p}\left(x^{\prime}(t)\right)\right)^{\prime}+f(x(t-\tau)) x^{\prime}(t-\tau)+\beta(t) g(x(t-\delta(t)))=e(t),
$$

where $\delta(t+T) \equiv \delta(t), \delta^{\prime}(t)<1$, and $\int_{0}^{T} \beta(s) d s \neq 0$ (the case of $\int_{0}^{T} \beta(s) d s>0$ will be considered in this section, and the other case, $\int_{0}^{T} \beta(s) d s<0$, can be studied in the same way). Then, according to Mawhin's continuation theorem, the following result is obtained with a similar process to Theorem 3.1.

Theorem 4.1 Assume that the following conditions hold:

$\left(C_{1}\right)$ There exist constants $r_{1}>0, r_{2}>0$, and $d_{1} \geq 0$ such that

$$
r_{1}|u|^{p-1} \leq|g(u)| \leq r_{2}|u|^{p-1} \text { and } u g(u)>0, \quad \forall|u|>d_{1} .
$$

$\left(C_{2}\right)$ There are constant $r_{3}>0$ and $d_{2} \geq 0$ such that $|f(u)| \leq r_{3}|u|^{p-1}, \forall|u|>d_{2}$.

$\left(\mathrm{C}_{3}\right)$ There is a constant $\varepsilon_{2}>0$ such that

$$
M:= \begin{cases}{\left[\frac{\left(\left|\beta^{-}\right|_{\infty}+\varepsilon_{2}\right) r_{2} T}{r_{1} \int_{0}^{T}\left(\beta^{+}+\varepsilon_{2}\right) d t}\right]^{\frac{1}{p-1}} 2^{\frac{2-p}{p-1}}<1,} & 1<p \leq 2, \\ {\left[\frac{\left(\left|\beta^{-}\right|_{\infty}+\varepsilon_{2}\right) r_{2} T}{r_{1} \int_{0}^{T}\left(\beta^{+}+\varepsilon_{2}\right) d t}\right]^{\frac{1}{p-1}}<1,} & p>2,\end{cases}
$$

where $\beta^{+}=\max _{t \in[0, T]}\{\beta(t), 0\}, \beta^{-}=\max _{t \in[0, T]}\{-\beta(t), 0\}$. 


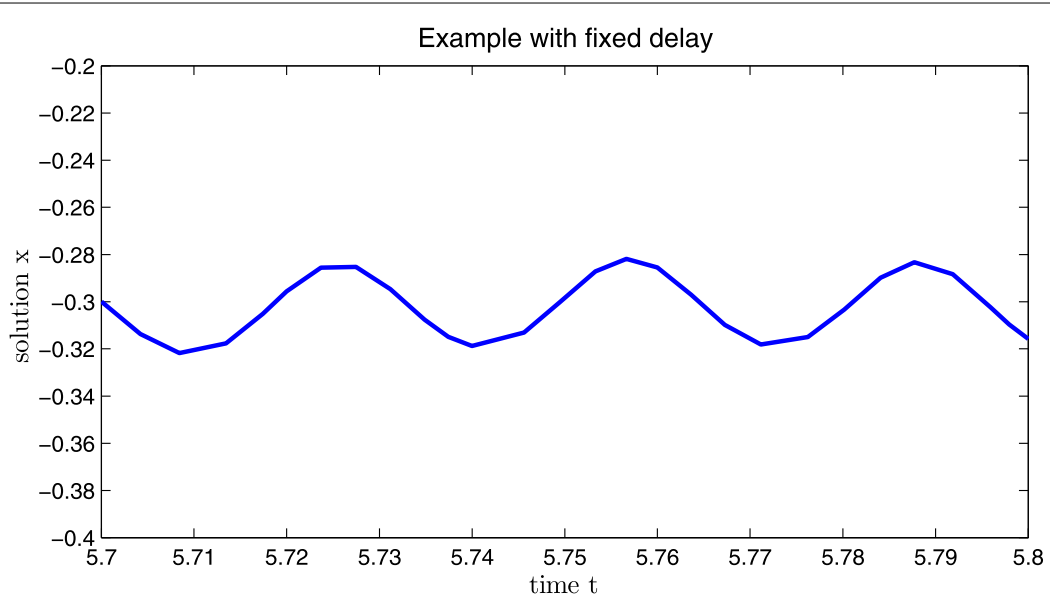

Figure 1 Phase diagram of the system for $\boldsymbol{\tau}=\mathbf{0 . 0 2}$.

Then (4.1) has at least one T-periodic solution if

$$
C_{p} r_{3} T^{p-1}+2^{p-1} r_{2} \theta|\beta|_{\infty} T^{p}<(1-M)^{p-1},
$$

where $\theta=\max _{t \in[0, T]} \frac{1}{1-\delta^{\prime}(\gamma(t))}$ and $\gamma(t)$ denotes the inverse of function $t-\delta(t)$.

\section{Example}

For convenience, as an application of Theorem 3.1, we consider the following example:

$$
\left(\varphi_{4}\left(x^{\prime}(t)\right)\right)^{\prime}+f(x(t-\tau)) x^{\prime}(t-\tau)+\left(\sin t+\frac{1}{2}\right) g(x(t-\tau))=\cos 200 t,
$$

where $p=4, f(t)=\frac{t^{2} \sin t}{500,000}, \beta(t)=\sin t+\frac{1}{2}, g(u)=\frac{u^{3}}{10,000}+1, e(t)=\cos 200 t$, then $F(u)=$ $\int_{0}^{u} f(s) d s=\frac{1}{500,000}\left(-u^{2} \cos u+2 u \sin u+2 \cos u-2\right)$, and $\beta_{\infty}=\frac{3}{2}$. So we can choose $m_{1}=$ $\frac{1}{10,000}, m_{2}=\frac{1}{5,000}, d=10 \sqrt[3]{10}, r=\frac{1}{500,000}, \varepsilon_{1}=\frac{1}{2}$ so that the conditions of Theorem 3.1 hold. Therefore, by Theorem 3.1 we find that (5.1) has at least one $\frac{\pi}{100}$-periodic solution, which can also be illustrated by numerical simulation.

By using MATLAB ${ }^{\odot} 7.12 .0$ (R2011a) toolkit: dde23, which can be used to solve delay differential equations with constant delays, $(5.1)$ is simulated on tspan $=[5.7,5.8]$ with history $=[0.5,-0.3]$ for $t \leq 0$ (see Figure 1$)$. It can be found from Figure 1 that the equation admits one periodic solution with periodicity 0.0314 , which is around $\frac{\pi}{100}$. Therefore, the results achieved in this paper are significant.

The authors declare that they have no competing interests.

Authors' contributions

All authors contributed equally to this work. All authors read and approved the final version of the manuscript.

\section{Author details}

'School of Mathematical Science, Yangzhou University, Yangzhou, 225002, China. ${ }^{2}$ Department of Engineering Science and Mechanics, Virginia Tech, Blacksburg, VA 24061, USA. ${ }^{3}$ College of Mathematics \& Statistics, Nanjing University of Information Science \& Technology, Nanjing, 210044, China. ${ }^{4}$ College of Mechanical Engineering, Beijing University of Technology, Beijing, 100124, China. 


\section{Acknowledgements}

This work is supported by the National Natural Science Foundation of China (NSFC) through grant Nos. 11302187,

$11271197,11172009,11372015$ and 61473340 . The authors are extremely grateful to the referees for their careful review of the manuscript and insightful comments, which helped to improve the paper greatly. The authors are also very grateful to Ms. Xiaojing Li for her original insightful ideas.

\section{Received: 8 October 2014 Accepted: 10 April 2015 Published online: 23 April 2015}

\section{References}

1. Amster, P, De Nápoli, PL, Mariani, MC: Periodic solutions for $p$-Laplacian-like systems with delay. Dyn. Contin. Discrete Impuls. Syst., Ser. A Math. Anal. 13, 311-319 (2006)

2. Gao, FB, Zhang, W: Periodic solutions for a p-Laplacian-like NFDE system. J. Franklin Inst. 348, 1020-1034 (2011)

3. Manásevich, R, Seedziwy, S: p-Laplacian and Liénard type equation. Rocky Mt. J. Math. 27, 611-617 (1997)

4. Manásevich, R, Mawhin, J: Periodic solutions for nonlinear systems with $p$-Laplacian-like operators. J. Differ. Equ. 45, 367-393 (1998)

5. Aizicovici, S, Papageorgiou, NS, Staicu, V: Multiple nontrivial solutions for nonlinear periodic problems with the p-Laplacian. J. Differ. Equ. 243, 504-535 (2007)

6. Gaines, RE, Mawhin, J: Coincidence Degree and Nonlinear Differential Equations. Springer, Berlin (1977)

7. Aizicovici, S, Papageorgiou, NS, Staicu, V: Periodic solutions for second order differential inclusions with the scalar p-Laplacian. J. Math. Anal. Appl. 322, 913-929 (2006)

8. Gao, FB, Lu, SP, Zhang, W: Periodic solutions for a Rayleigh type $p$-Laplacian equation with sign-variable coefficient of nonlinear term. Appl. Math. Comput. 216, 2010-2015 (2010)

9. Gao, FB, Lu, SP: Periodic solutions for a Rayleigh type equation with a variable coefficient ahead of the nonlinear term. Nonlinear Anal., Real World Appl. 10, 254-258 (2009)

10. Cheung, HM: On p-Laplacian equations with deviating arguments. Master thesis, The University of Hong Kong (2009) 